\title{
Révolutions et Républiques : la France contemporaine
}

\section{Louis Hincker}

\section{(2) OpenEdition \\ 12 Journals}

\section{Édition électronique}

URL : https://journals.openedition.org/ahrf/7753

DOI : 10.4000/ahrf.7753

ISSN : 1952-403X

Éditeur :

Armand Colin, Société des études robespierristes

\section{Édition imprimée}

Date de publication : 1 décembre 2006

Pagination : 151-153

ISSN : 0003-4436

\section{Référence électronique}

Louis Hincker, «Révolutions et Républiques : la France contemporaine », Annales historiques de la Révolution française [En ligne], 346 | Octobre/Décembre 2006, mis en ligne le 10 juillet 2008, consulté le 23 avril 2022. URL : http://journals.openedition.org/ahrf/7753; DOI : https://doi.org/10.4000/ahrf. 7753

Ce document a été généré automatiquement le 23 avril 2022.

Tous droits réservés 


\title{
Révolutions et Républiques : la France contemporaine
}

\author{
Louis Hincker
}

\section{RÉFÉRENCE}

Pierre Lévêque, Révolutions et Républiques : la France contemporaine, Dijon, Éditions Universitaires de Dijon, 2005, 350 p., ISBN 2-915552-35-5, $20 €$.

1 Les publications de recueils d'articles sont peu nombreuses ; c'est un tort. Elles rendent compte d'un cheminement, d'une méthode, d'un style, elles soulignent le caractère précurseur, parfois quasi définitif, de travaux qui retrouvent une nouvelle vigueur. Telle est la facture de ce recueil d'études de Pierre Lévêque, professeur émérite de l'Université de Bourgogne, qui rassemble des textes d'un spécialiste reconnu de l'histoire politique et sociale d'un long $\mathrm{XIX}^{\mathrm{e}}$ siècle français prenant racines dans l'événement révolutionnaire et continuant de conditionner ce que l'auteur a choisi de désigner sous l'expression de "France contemporaine ». De 1966 à 2004, ces textes ont le plus souvent eu pour cadre soit des colloques, soit - et notamment pour les plus récents - des dictionnaires ou encyclopédies ayant pour fonction d'offrir de denses et concises contributions sur l'histoire des différentes révolutions et républiques qui se sont succédé en cascade depuis 1789. C'est dire que Pierre Lévêque s'affirme comme un spécialiste de la synthèse, particulièrement salué à ce titre par Alain Corbin dans sa préface à cet ouvrage. On notera une attention particulière portée par l'auteur, et maintes fois répétée, au rôle des milieux populaires dans cette histoire politique mouvementée: acteurs prépondérants des Cents-Jours, électeurs novices de 1848, éléments à la base de la Libre Pensée, terreau de la «République démocratique et sociale » dont Pierre Lévêque souligne le caractère prégnant et l'histoire longue.

2 Certains des articles s'imposent comme des démonstrations magistrales où le lecteur suit pas à pas les ressorts d'une démarche rigoureuse. Deux particulièrement nous paraissent être les véritables points d'orgue de la méthode de l'auteur, celui sur « La 
Patente, indicateur de croissance économique différentielle au XIXe siècle ?» et celui sur "Vigne, religion et politique en France aux $\mathrm{XIX}^{\mathrm{e}}$ et $\mathrm{XX}^{\mathrm{e}}$ siècles » : prudence, goût pour les questions plutôt que pour les conclusions abusives, mais au service d'une synthèse précise de phénomènes saisis à l'échelle nationale, grâce à une connaissance des sources de première main acquise à l'école d'une monographie régionale - grand œuvre et thèse d'État de l'auteur : La Bourgogne de la Monarchie de Juillet au Second Empire, ARNT Lille 3, 1980 -, une de ces monographies de référence pour toute histoire de la France du XIX ${ }^{e}$ siècle. L'évolution de la répartition géographique des patentés sur le territoire, résumée par une série de coupes de 1827 à 1913, permet de saisir l'inégale densité des activités commerciales et industrielles (montant total du principal de l'impôt multiplié par six, nombre total de cotes par trois, et valeur de la cote moyenne par deux) au profit finalement assez tardif de la capitale et des grandes villes après avoir, jusqu'au cœur du Second Empire, connu un développement équilibré sur l'ensemble du territoire. Pierre Lévêque est un spécialiste de l'histoire des régions viticoles et sa contribution fait un sort à cet acteur récurrent de la geste révolutionnaire française : le vigneron. Loin d'être une donnée intangible de l'histoire de la France contemporaine, l'orientation à gauche des régions viticoles n'est finalement pas très ancienne ni vraiment durable, et certainement pas mécanique : elle culmine au temps du radicalisme au tournant du siècle avec pour socle la défense de la propriété, une indifférence religieuse - voire un anticléricalisme - mais une grande méfiance vis-à-vis de l'idéologie révolutionnaire. Cette relative et tardive cohérence des options politiques - en même temps qu'une fragilité récurrente face à la conjoncture économique qui amène le vigneron à réagir en désignant les pouvoirs établis comme responsables de ses difficultés -, ne doit pourtant pas cacher la diversité structurelle et de longue durée de la condition du vigneron: importance de la grande et moyenne propriété en faire-valoir indirect, classe moyenne des petits exploitants indépendants, saisonniers. Et Pierre Lévêque de conclure à l'autonomie des phénomènes politiques et de se méfier des corrélations trop facilement admises.

3 Sur ce primat du politique - derrière lequel se range Pierre Lévêque dans un texte qui sert par ailleurs d'avant-propos: "Pour une nouvelle histoire politique» - on redécouvrira dans ce recueil d'études un autre petit bijou, devenu un classique, longtemps la seule véritable enquête documentée sur les rapports entre «La Libre Pensée et le socialisme (1889-1939) quelques points de repères » parue dans la revue Le Mouvement Social en 1966. Pierre Lévêque y dresse un tableau très référencé des principales organisations nationales d'un mouvement tout à la fois et paradoxalement populaire et minoritaire, convoité par la SFIO et le Parti Radical, oscillant entre une stricte Libre Pensée anticléricale et une aspiration à une Libre Pensée sociale, finalement déçu par le Front Populaire car préférant ne rien sacrifier aux antagonismes idéologiques toujours premiers et au-dessus des intérêts de classe.

Dans la droite ligne de ce premier travail pionnier et précurseur, Pierre Lévèque s'est affirmé durant ces dernières décennies comme un historien de référence de ce qu'il a pour habitude d'appeler les "forces politiques" sur lesquelles il a réalisé une vaste fresque chez Armand Colin en trois volumes parus en 1992, 1994 et 1997. Plusieurs articles de ce recueil témoignent de ce privilège accordé à ces phénomènes qualifiés d'« éminemment politiques": "organisations", "courants ", "culture politique ", « militants » et " dirigeants ", « avant-garde », «traditions », « héritages ", « modèles ", "legs", "mémoires", " réminiscences», « résurgences", "traces profondes", «étapes de développement» et autres "attitudes habituelles», «fonctionnement 
normal des institutions ", "décisions marquantes ", " actions persévérantes ", "prévisions", "tactique", "efficacité immédiate», ou encore par contraste " réactions éventuelles du corps social ", "vote protestataire ", " mauvaise passion ", "déceptions", "hostilités", "vide politique relatif», «expression spontanée», " folklore ", " anarchie ", " groupements clandestins » " parenthèse brève et stérile ". Avouons que nous sommes restés quelque peu perplexe devant ce vocabulaire interprétatif dont nous ne citons ici que quelques- unes des formules et expressions saillantes et qui foisonne particulièrement dans les deux textes du recueil s'offrant comme récits des différents épisodes de la Seconde République, de laquelle Pierre Lévêque est un éminent spécialiste. Mais qu'est-ce à dire que de qualifier les années 1848-1851 de "crise révolutionnaire " ou encore d'«ébranlement et restauration de l'ordre social ", pour reprendre les deux titres de ces articles parus en 1998 à l'occasion du cent-cinquantenaire de la Révolution de 1848 ? En l'absence de toute réflexion sur la nature et les origines du vocabulaire mobilisé pour l'analyse, à grand renfort de guillemets, tantôt pour signifier une (la ?) langue d'une époque, tantôt pour établir une complicité supposée avec le lecteur, et le plus souvent par le seul truchement du style indirect libre, ne risque-t-on pas d'aboutir à la promotion de la langue du libéralisme politique comme langue de l'interprétation scientifique au service d'une histoire advenue et aux dépens des expériences inédites et éphémères dont le sens s'est en partie perdu? Grave question qui est celle du discours explicatif adéquat pour qualifier ces moments de discontinuité politique que nous nommons « révolutions ». Et juin 1848 aura été «terrible» (p. 268) autant que la Commune «douloureuse» (p. 84). Loin de la prudence et du doute méthodique qui fait tout le suc des articles certes pointus mais fondamentaux du recueil, les vastes panoramas sur les années 1848-1851 mais aussi sur la gauche et l'idée révolutionnaire, les Républiques, ou encore l'idée d'égalité, nous semblent par trop dans les pas de grands auteurs - Tocqueville en tête -, ou encore parfois des rapports des préfets, cités pour corroborer une démonstration à l'origine comme au final tirée de leurs propres analyses. Cela n'enlève rien à l'importance d'une telle publication, dont le genre, répétons-le, devrait être plus fréquent et au service des débats historiographiques. 